Recherches en didactique des langues et des cultures

Les cahiers de l'Acedle

$15-2 \mid 2018$

Quelles médiations en didactique des langues et des cultures?

\title{
Vers une grammaire scientifique et didactique en classe de fle : L'exemple de la préposition en français
}

\section{Assiakh Sofiane et Sadi Nabil}

\section{OpenEdition}

\section{Journals}

Édition électronique

URL : https://journals.openedition.org/rdlc/3073

DOI : $10.4000 /$ rdlc.3073

ISSN : 1958-5772

Éditeur

ACEDLE

Référence électronique

Assiakh Sofiane et Sadi Nabil, « Vers une grammaire scientifique et didactique en classe de

fle : L'exemple de la préposition en français », Recherches en didactique des langues et des cultures [En

ligne], 15-2 | 2018, mis en ligne le 02 juin 2018, consulté le 21 septembre 2021. URL : http:// journals.openedition.org/rdlc/3073; DOI : https://doi.org/10.4000/rdlc.3073

Ce document a été généré automatiquement le 21 septembre 2021.

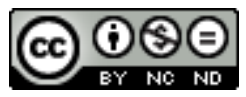

Recherches en didactique des langues et des cultures is licensed under a Creative Commons AttributionNonCommercial-NoDerivatives 4.0 International License 


\title{
Vers une grammaire scientifique et didactique en classe de fle : L'exemple de la préposition en français
}

\author{
Assiakh Sofiane et Sadi Nabil
}

\section{Introduction}

1 Dans la tâche enseignement/ apprentissage du FLE, il arrive que les enseignants fassent appel à des ouvrages de grammaire afin de pouvoir élaborer leurs fiches pédagogiques, se rappeler une règle, combler une lacune ou chercher des informations complémentaires. Les apprenants n'hésitent pas à leur tour à se référer à ce type d'ouvrages afin de mieux appréhender un point de langue qu'ils ignorent ou qu'ils n'ont pas bien retenu lors d'un cours de grammaire. Mais il arrive également que l'enseignant tombe, en élaborant son cours de grammaire, sur des grammaires dont le contenu est plus ou moins lacunaire d'un point de vue linguistique (contenu non-valide, incohérent, polysémique, etc.) ou/et didactique (incomplet, formel, illisible, abstrait, etc.) ${ }^{1}$. Cela devrait sans doute influer négativement sur la qualité de leurs discours et ipso facto sur la maitrise des apprenants du point de langue en question. De ce fait, nous pensons que la linguistique peut pallier les lacunes de la grammaire dans l'enseignement du FLE, ce qui serait une sorte de réconciliation entre deux disciplines longtemps opposées et, selon l'expression de A. BERRENDONNER (cité par CUQ J-P, 1996: 25), antagonistes vu le contraste des rôles assignés au linguiste et au grammairien: «le grammairien, abusé par sa démarche préscientifique, aurait été limité à un rôle morale et perspectif; le linguiste dans l'impartialité de sa démarche scientifique, aurait eu seul vocation de démêler l'objectif du subjectif » (Ibid.). Notre réflexion peut également être considérée comme un retour aux ambitions de la linguistique appliquée dans les années 1950, vouées par la suite à l'échec avec l'apparition de la didactique en 1970 (PUREN Ch., 2008). Notre objectif est de démontrer que les 
fondements de la linguistique pourraient constituer un adjuvant aux discours grammaticaux que nous supposons plus ou moins lacunaires à cause de leur qualité et la manière dont ils sont présentés.

\section{De la grammaire à la linguistique}

2 Indéniablement, l'enseignement de la grammaire a constamment été une forte préoccupation autant pour les méthodologues que pour les enseignants de langue. En effet, bien qu'il ne se soit vraiment imposé en classe de langue qu'à partir du XVIII ${ }^{\text {ème }}$ siècle $^{2}$, son enseignement remonte à l'époque de Platon et d'Aristote (J-P. CUQ, 1996: 08). Dans une perspective pédagogique, cette composante linguistique, dont on a souvent vanté les mérites comme savoir facilitant l'apprentissage d'une langue donnée, a été souvent considérée comme étant un savoir difficile à faire acquérir vu sa complexité d'une part et son caractère autoritairement normatif et injonctif d'une autre part. A ce niveau, la grammaire comme discipline tâche de décrire, en faisant de la dichotomie correct /incorrect son principal critère du jugement, les règles auxquelles on doit se soumettre pour pouvoir bien parler et écrire.

3 Vers le XX siècle, le champ des investigations linguistiques s'ouvrait sur de nouvelles voies et influençait, par conséquent, le devenir des théories ayant la langue comme objet d'étude pour donner naissance à la linguistique, discipline qui se démarque par sa rigueur scientifique, sa démarche analytique et sa terminologie élaborée. En effet, à cette époque :

[...] se développait une véritable discipline autonome, attentive à la définition de son objet, soucieuse de promouvoir un appareil conceptuel, des principes méthodologiques et des procédures d'analyse avec les exigences de rigueur et de cohérence qui sont familières à la plupart des sciences modernes: la linguistique. (ARRIVE M. \& GADET F., cité par PETIOT G., 2000 : 18).

4 En effet, héritée de la tradition grammaticale dont le caractère était normatif et prescriptif, la linguistique avait pour principale vocation de dissocier l'objectif du subjectif en se fixant comme objectif principal «l'étude scientifique du langage » (J. DUBOIS et all, 1999 : 285). A. MARTINET explique qu'une discipline, à l'instar de la linguistique, est dite scientifique «lorsqu'elle se fonde sur l'observation des faits, et s'abstient de proposer un choix parmi ces faits au nom de certain principes esthétiques et muraux. «Scientifique » s'oppose donc à «prescriptif».» (2005: 06). En effet, dans sa description des faits de langue, le linguiste adopte une démarche particulièrement explicative qui consiste à analyser/ déterminer le fonctionnement régissant la langue et le langage et les rapports existant entre leurs différentes unités. Une démarche tout à fait différente de celle adoptée par le grammairien qui se focalisent plutôt sur le listage des lois de fonctionnements d'une langue donnée sans toujours les expliquer, ce qui fait de sa description une organisation fondée sur un ensemble d'assertions dogmatiques (D. GIRARD, 1972:15) qui, d'une manière prescriptive (correct/ incorrect, dites/ ne dites pas), décrète les lois régissant le fonctionnement interne d'une langue donnée « qu'est-ce en effet qu'une grammaire sinon une grille de lecture qui, projetée sur les énoncé de la langue, nous révèle comment ils sont, doivent ou devraient être construits. » (RIEGEL M. \& PELLAT J-C. \& RIOUL R., 2009 : XV).

5 Indubitablement, la naissance de la linguistique appliquée vers les années 1950 a constitué un rétablissement des tâches assignées aux linguistes et aux grammairiens et une incarnation de la contribution de la linguistique scientifique à l'enseignement des langues en général et de la grammaire en particulier. J-L. CHISS pense que «[...] le terrain 
de l'enseignement de la langue soit le lieu par excellence de validation de la linguistique, qu'on ne puisse séparer la recherche concernant l'efficace de la linguistique sur la rationalisation pédagogique» (2016:19). La linguistique se voit ainsi révolutionner le champ de l'enseignement de la grammaire à travers l'application des théories structuralistes, en particulier le distributionnalisme, en classe de langue (la méthodologie audio orale). Cette rupture épistémologique constitue à notre sens une réconciliation entre deux disciplines, linguistique et grammaire, dont la séparation engendre la stérilité de la première et le dogmatisme de la deuxième.

6 Dans le présent article, nous nous intéresserons à la disposition des discours linguistiques d'être impliqués dans unenseignement de langue, de grammaire en l'occurrence. A ce niveau, le discours linguistique est envisagé comme objet d'enseignement et non pas un outil au service de l'enseignement, comme c'est le cas dans la linguistique appliquée. Ainsi, nous passerons d'une linguistique appliquée à une linguistique applicable à l'enseignement/ apprentissage du FLE et ce grâce à un concept novateur qui est la valeur d'applicabilité (M-E. DAMAR, 2009).

\section{La valeur d'applicabilité d'un discours linguistique}

Elaborée principalement par M-E. DAMAR, la valeur d'applicabilité constitue « la tendance d'une théorie linguistique à être plus ou moins transposable pour l'enseignement » (2009: 101), c'est-à-dire le potentiel d'un contenu d'être enseigné dans une classe de langue. Il s'agit d'un ensemble de critères, puisés de la philosophie des sciences, qui évaluent la scientificité linguistique et la didacticité des contenus destinés à l'enseignement/ apprentissage.

8 Partant de l'hypothèse selon laquelle la linguistique peut pallier les lacunes de la grammaire, nous avons décidé d'emprunter le modèle théorique de M-E DAMAR, étant donné qu'il présente des convergences importantes avec le travail de recherche auquel nous nous sommes livrés, un travail dans lequel nous avons tenté de mettre en lumière le soutien que les recherches linguistiques pourraient apporter à l'enseignement/ apprentissage de la grammaire en classe de FLE. Une telle réflexion est d'ailleurs mise en exergue par d'autres linguistes et didacticiens à l'instar de S. CANELAS-TREVISI pour qui "Les emprunts de la linguistique scientifique devaient venir modifier la grammaire de l'école en la rendant plus efficace.» (2009: 56) et S. P. CORDER qui admet que «les théories linguistiques nous fournissent la plupart des concepts et du vocabulaire que nous utilisons pour parler de ce que nous enseignons » (cité par J-L CHISS, 2016 :17).

\section{Méthodes et matériel}

Afin de confirmer ou d'infirmer notre hypothèse, nous avons d'abord constitué un corpus de recherche de trois des grammaires les plus usitées par les enseignants du FLE en Algérie, à savoir Le Larousse (2008), Le Robert \& Nathan (1995) et Le Bescherelle (2012), ainsi que trois discours de linguistes traitant des prépositions de la langue française: D. LEEMAN (2008), G. GROSS (2006) et J. CERVONI (1990). Afin de pouvoir déceler les avantages et les lacunes caractérisant le contenu de ces discours, nous avons passé en revue, pour chaque précepte avancé, le potentiel explicatif, la cohérence, la validité et l'économie sur le plan de la scientificité linguistique, et, la globalité, l'intégration du sens, 
la lisibilité et l'intelligibilité sur le plan didactique ${ }^{3}$. De cette manière, nous avons pu établir des constats qui, moyennant une grille d'analyse (voir ci-dessous), nous ont permis d'adopter des éléments de réponse à la problématique posée.

\section{Examen des grammaires}

Nous entamerons notre analyse par un examen de grammaires Le Bescherelle, Le Larousse et Le Robert \& Nathan, afin de mettre en lumière la scientificité linguistique et la didacticité de leurs contenus quant à la description de la préposition et des locutions prépositives.

\section{Les critères de scientificité linguistique}

Attribuer à un discours la scientificité comme caractère, c'est d'en évaluer la satisfaction/ transgression de certains principes intrinsèques à l'activité scientifique. En effet, une activité intellectuelle ne saurait remporter sa scientificité que lorsque «[...] elle respecterait certains critères ou certaines façons reconnues par la communauté des chercheurs comme étant légitimes [...] 》 (MATHIEU A., 2011: 56). Il s'agit en effet d'un ensemble de critères épistémologiques évaluant la crédibilité, la véracité et la fiabilité des discours. Pour notre part, nous retenons cette réflexion pour l'adapter aux discours grammaticaux. En grammaire, ainsi qu'en linguistique, un discours dit scientifique devrait essentiellement être explicatif, cohérents, valide et économique.

\section{Explication Vs catalogue}

Un discours dit scientifique est un discours qui, outre le catalogage de ses préceptes, tient également à relater méticuleusement le fonctionnement, les principes et les caractéristiques de l'information avancée. D'un point de vue linguistique, un discours ne pourrait être qualifié de scientifique que s'il jouit d'un potentiel explicatif qui lui attribue plus d'intelligibilité et de compréhensibilité. Accéder au mécanisme régissant le fonctionnement d'un point de langue donné serait bien entendu beaucoup plus avantageux et bénéfique pour l'apprenant que d'en apprendre par cœur une liste de règles ou d'emplois. Comprendre une règle à partir d'une explication rigoureuse est, selon l'expression de M-E DAMAR, «moins coûteux cognitivement que retenir en extension un catalogue d'emplois » (2009: 107).

13 L'analyse de l'explication et du catalogage dans les trois grammaires constituant notre corpus révèle qu'elles constituent des discours grammaticaux plus ou moins normatifs de par la manière dont les faits grammaticaux sont présentés. La tendance la plus dominante est ainsi d'établir des listes et des catalogues d'emplois, des règles de fonctionnement et des caractéristiques lexicosémantiques et morphosyntaxiques de la préposition (Le Bescherelle: 211-213 /Le Robert \& Nathan: 223-227) sans lui fournir des explications rigoureuses pour rendre son apprentissage plus aisé. L'apprenant est ainsi appelé à mémoriser les règles grammaticales au lieu d'en comprendre le fonctionnement afin de pouvoir généraliser ses acquis sur d'autres contextes.

Dans le Bescherelle par exemple (\$293), les types de prépositions sont évoqués sous forme d'un inventaire des prépositions simples et des locutions prépositionnelles les plus usitées en langue française. Quant à leurs caractéristiques morphologiques, elles sont passées au second plan. Comme conséquence, l'apprenant pourrait considérer tout 
groupe de mots obéissant à la syntaxe Pré $+N / V+$ Pré comme étant une locution prépositive.

Dans un passage consacré aux cas de non-répétition des prépositions, Le Larousse dresse une liste desquatre principaux cas sans expliquer que dans l'énoncé « En mon âme et conscience, je le crois coupable", la non-répétition de la préposition "en» est due à la présence d'une conjonction de coordination, ce qu'on remarque aussi dans les trois autres cas. A partir de cette explication, l'apprenant serait en mesure de retenir un seul principe et de le généraliser sur les autres cas au lieu de devoir retenir toute une liste d'emplois.

\section{Cohérence Vs contradiction}

La scientificité d'un discours se rapporte également à son degré de consistance et de cohérence. Un discours destiné à l'enseignement doit être exempt de toute contradiction et antilogie, autrement, il pourrait nuire à la compréhension de l'apprenant. En grammaire, un énoncé ou un exemple qui réfute une règle précédemment établie pourrait constituer une source de confusions pour l'apprenant. La cohérence serait ainsi assimilée à un «[...] principe de non contradiction. La contradiction est une relation entre deux éléments ou entre deux énoncés dont l'un soutient ce que l'autre nie. [...] 》 (DAMAR M-E., 2009: 97).

Le Bescherelle, Le Larousse et Le Robert \& Nathan reposent sur des discours grammaticaux incohérents compte tenu des contradictions internes qui caractérisent leurs contenus. Dans Le Bescherelle par exemple, le fait de dresser une liste des multiples compléments qu'une préposition permet d'introduire (\$293, p212) et de fournir un exemple qui ne constitue nullement un complément mais plutôt un attribut, révèle la contradiction de l'auteur avec ce qu'il avance : « ils me prennent pour un naïf ».

Selon Le Larousse, la préposition serait « un mot invariable qui joint un nom, un pronom, un adjectif, un infinitif ou un gérondif à un autre terme [...] (P. 122). L'incohérence de cette définition réside dans le fait d'employer un terme pour désigner une réalité qui ne correspond pas aux exemples fournis. En effet, le fait de souligner que la préposition est un «mot » et de fournir ultérieurement des exemples comme «à cause de, à force de, audessous de..., etc. » (Ibid.) pour mettre l'accent sur les locutions prépositives révèle une contradiction de l'auteur. Une contradiction qui pourrait engendrer des confusions chez l'apprenant pour qui la préposition constitue un seul mot, ce qui fait de l'énoncé «Il lui envoya la main en guise de remerciement" un énoncé construit à partir de deux prépositions : en et de, alors que l'on a affaire à une locution prépositive en guise de.

Dans Le Robert \& Nathan (p.223, §457), on note que dans une phrase, une préposition peut être suivie de mots de différentes natures, entre autres un gérondif. Alors que dans un autre paragraphe (\$459, p224), il souligne que dans la phrase «il est parti en sifflant», la préposition est suivie d'un participe présent et que la préposition en forme avec ce participe un gérondif. Donc, ce n'est pas le mot (le participe) qui vient après la préposition en qui constitue le gérondif, mais plutôt, la préposition jointe à ce participe. En effet, la règle, telle qu'elle est présentée dans le premier paragraphe et illustrée et expliquée dans le second, révèle une contradiction qui pourrait induire en erreur l'apprenant. 


\section{La validité}

Bescherelle, Le Larousse, Le Robert \& Nathan), un groupe de mots qui établit un lien entre deux mots ou deux groupes de mots (Le Bescherelle, Le Larousse) et qui permet d'introduire un mot ou un groupe de mots (Le Larousse, Le Robert \& Nathan). La polysémie et la plurivocité de la notion de mot et de l'expression groupe de mots pourrait constituer une source de confusion pour l'apprenant. Ainsi, la notion de mot peut renvoyer parfois à une seule unité graphique ou phonétique (arbre) ou à plusieurs (pommes de terre). L'expression groupe de mots pourrait, elle aussi, renvoyer à une seule unité sémantique (à force de) ou à plusieurs (à huit heures). De ce fait, l'apprenant pourrait prendre tout ce qui constitue pour lui un groupe de mots pour une locution prépositive. Il en va de même des expressions sens stable/ multiple (Le Bescherelle :212), seul/ multiples rapports (Le Larousse: 123), sens précis/ variés (Le Robert \& Nathan: 225), auxquelles nous préférons substituer les deux 
qualificatifs «monosémique/ polysémique ». L'idée serait ainsi exprimée à partir d'un seul procédé explicatif, plus laconique et plus intelligible.

\section{Les critères de didacticité}

Qualifier un discours de scientifique d'un point de vue linguistique ne signifie pas qu'il constitue un contenu enseignable. En effet, un enseignement doit être avant tout une transposition d'un savoir jugé scientifique, tout en tenant compte des besoins des apprenants et de leur profil pédagogique. A cette transposition, viennent s'imbriquer d'autres critères auxquels les contenus grammaticaux doivent répondre positivement afin d'assurer leur didacticité. Un discours dit didactique serait également un discours global, intégrant du sens, lisible et intelligible.

\section{Modèle global Vs modèle modulaire}

Sur le plan descriptif, un discours grammatical qui cerne toutes les dimensions d'un point de langue donné (morphologique, syntaxique et sémantique) est plus approprié didactiquement qu'un autre qui se limite à certains de ses aspects. Une connaissance partielle d'un point de grammaire engendra automatiquement une maitrise partielle de ses différents modes d'emploi. L'idéal serait d'amener l'apprenant à «[...] appréhender la manière dont s'organise le système en son entier » (M-E. DAMAR, 2010 : 119-120).

D'un point de vue didactique, Le Larousse et Le Robert \& Nathan offrent des contenus grammaticaux globaux de par les aspects développés dans la description des faits grammaticaux (morphologie, syntaxe, sémantique). Le discours du Bescherelle constitue, par contre, un discours plus ou moins modulaire, compte tenu de la négligence de l'aspect sémantique des prépositions.

\section{Intègre du sens / permet de construire du sens}

L'objectif ultime de tout enseignement de langue est d'amener l'apprenant à développer ses compétences de compréhension et de production du sens afin de pouvoir communiquer dans la langue cible. De ce fait, un discours grammatical qui favorise les aspects syntaxique et morphologique du point de langue décrit au dépens de celui sémantique ne saurait aider l'apprenant ni à comprendre ce point ni à l'employer pour communiquer en français. Ce critère est évalué sur deux niveaux relatifs à la reconnaissance et la production des formes : «l'intégration du sens peut se manifester à deux niveaux[...] la théorie qui prend moins en compte le sens à celle qui permet de le produire. Il faut idéalement que l'explication permette à la fois de reconnaitre les emplois [...] mais aussi de produire lesdits emplois avec un risque d'erreur minimal. » (M-E. DAMAR, 209 : 103-104).

Les trois grammaires analysées offrent des discours didactiques de par le recours systématique au sens dans la description et l'explication des faits. Cependant, en négligeant le sémantisme des prépositions et leur polysémie, les grammaires Le Bescherelle et Le Robert \& Nathanne sauraient amener l'apprenant à produire du sens, étant donné qu'elles ne lui donnent pas l'outil indispensable qui lui permet de produire des énoncés exprimant des rapports spatiaux, temporels, causaux, etc., ce qui l'aurait sans doute sensibilisé du bon usage des prépositions sur le plan sémantique. 


\section{La lisibilité}

31 Considéré comme étant à la fois un critère d'évaluation de la scientificité et de la didacticité des discours, la lisibilité désigne "l'aptitude d'un texte, généralement imprimé, à être lu avec aisance et efficacité » (F. RICHAUDEAU cité par BLANQUET M-F, 2009 : 17-18). ME DAMAR (2009) situe ce critère sur deux niveaux plus ou moins convergents : le niveau formel et le niveau matériel. Le premier est une réalité d'ordre linguistique : les mots ne doivent pas dépasser trois syllabes, le métalangage doit être défini, limité et imagé pour ne pas nuire à la compréhension. Les phrases ne doivent pas dépasser vingt mots et doivent être d'une syntaxe simple $(\mathrm{S}+\mathrm{V}+\mathrm{C})$. Quant au deuxième, il renvoie plutôt à une réalité d'ordre typologique : la façon dont le discours grammatical est présenté doit être schématisée en recourant à titre d'exemples à des tableaux et des schémas et tout ce qui pourrait mieux organiser les informations transmises.

Sur le plan formel, les mots employés dans les trois discours analysés sont généralement courts avec une graphie qui ne dépasse pas trois syllabes. Quant au métalangage, il est limité mais souvent indéfini (sauf pour les prépositions et les locutions prépositives). Au niveau des phrases, Le Bescherelle etLe Larousseoffrent des discours dont les phrases sont courtes (elles ne dépassent pas vingt mots) et la syntaxe est simple $(\mathrm{S}+\mathrm{V}+\mathrm{C})$. Quant au Robert \& Nathan, les phrases employées sont souvent longues (plus de vingt mots dans certains cas) et complexes (construites souvent à partir d'un pronom relatif).

Sur le plan matériel, les procédés explicatifs déployés dans Le Larousse sont souvent d'ordre linguistique sans aucun recours aux schémas ou aux tableaux pour mieux illustrer et structurer les informations avancées. Dans Le Bescherelle (p.212) et Le Robert \& Nathan (pp. 226-227), le souci d'organisation et de schématisation est remarquablement apparent dans l'utilisation des tableaux afin de faciliter la compréhension à l'apprenant.

\section{L'intelligibilité}

L'une des caractéristiques d'un discours dit didactique est son intelligibilité qui se traduit par la susceptibilité de son contenu d'être appréhendé et assimilé aisément par l'apprenant. Pour qu'un discours puisse être qualifié d'intelligible, il doit répondre à quatre sous-critères :

\section{La progressivité}

35 Un discours serait intelligible s'il est «fractionnable, en liaison étroite avec une progression didactique» (M-E DAMAR, 2010 : 124). A ce niveau, nous nous référons à une conception d'un contenu grammatical qui, tout en gardant sa cohérence, peut être enseigné d'une manière croissante et évolutive, autrement dit, un contenu dont les aspects pourront être enseignés encrescendo, du simple au complexe et du facile au difficile.

$\mathrm{Au}$ niveau de leur intelligibilité, les trois grammaires analysées présentent des discours grammaticaux thématiquement progressifs et fractionnables. Les différents aspects convoqués dans la description des prépositions sont présentés d'une +manière fractionnable, obéissant dans la majorité des cas à une progression logique. 


\section{La parcimonie}

37 Le critère de parcimonie se rapporte, selon M-E DAMAR (2009) au nombre de concepts et de notions déployés dans la description d'un point de langue donné. Un nombre qui devrait de préférence être réduit pour que l'apprenant puisse appréhender aisément les informations avancées. L'utilisation systématique de concepts et de mots clés est fort souhaitable afin d'éviter l'abondance terminologique.

38 Toutes les grammaires analysées offrent des discours parcimonieux de par le nombre réduit des concepts évoqués dans la description de la préposition.

\section{La concrétude}

39 Un discours didactique doit indispensablement interpréter le réel et cela ne saurait se réaliser qu'en s'éloignant de toute abstraction qui pourrait entraver l'apprenant dans son apprentissage, le concret étant logiquement beaucoup plus facile à retenir que l'abstrait. Une raison pour laquelle nous préférerons les discours dont on emploie des concepts concrets plutôt à d'autres qui inondent l'apprenant d'abstractions.

40 Le Bescherelle, Le Larousse et Le Robert \& Nathan sont des grammaires dont le discours est caractérisé par la concrétude de ses énoncés et de ses concepts. Ainsi, pour décrire la préposition, ces grammaires recourent à des concepts souvent incarnée à partir d'exemples de la vie ordinaire. Par ailleurs, une nuance d'abstraction est inéluctablement présente étant donné que, contrairement aux substantifs par exemple, dont le référent est aisément identifiable, la préposition est dépourvue de tout référent ou objet qui l'incarne dans la vie tangible; elle renvoie ainsi à des constructions mentales et à des réalités intrinsèquement abstraites (positionnement dans le temps, dans espace, le but, etc.)

\section{Emploi d'images et de métaphores incarnant la vie quotidienne de l'apprenant}

41 Dans ce critère, nous privilégierons les discours où l'on se sert d'images, de métaphores, métonymies, etc. comme supports d'illustrations des contenus enseignés. L'objectif est de rendre l'information plus concrète pour l'apprenant, d'autant plus que le visuel constitue un pont entre l'abstrait et le concret: "Le visuel, le concret, se prête mieux à leur compréhension et leur mémorisation. Il n'est pas pour autant question de barrer ou de réduire les notions abstraites. Mais il est recommandé de les accompagner de comparaisons, d'exemples, pourquoi pas de métaphores pour augmenter leur force ». (F. RICHAUDEAU cité par M-E DAMAR, 2009 : 108).

42 Ce procédé explicatif est totalement inusité dans les grammaires analysées. Aucun auteur n'utilise des images pour illustrer les rapports sémantiques que peut établir une préposition, mais ils se contentent plutôt de la langue comme principal support d'explication.

\section{Examen des discours linguistiques}

43 Nous tâcherons à présent d'évaluer la scientificité linguistique et la didacticité de trois discours linguistiques (D. LEEMAN, G. GROSS, J. CERVONI) afin de mettre en lumière leur 
degré d'applicabilité à l'enseignement/ apprentissage de la préposition et des locutions prépositives.

\section{Les critères de scientificité linguistique}

\section{Explication Vs catalogue}

L'examen des discours linguistiques a révélé que leurs contenus se caractérisent par une démarche explicative qui écarte toute forme de listage et de prescription de règles. Les points de langue sont ainsi relatés à partir d'explications rigoureuses, détaillées et illustrées par des exemples intelligibles.

D. LEEMAN (2008) propose un discours qui dépassent le simple listage de règles, un discours dans lequel elle englobe tout ce qui est prépositionnel. Sur le plan sémantique par exemple, elle établit trois classes de prépositions: monosémiques, polysémiques, incolores ou vides. Il en va de même avec le discours de G. GROSS (2006: 02) dont les locutions prépositives sont exposées à partir de leurs procédés de formations. En effet, une transposition didactique de ces derniers points permettrait sans doute à l'apprenant d'appréhender le sémantisme des prépositions et de reconnaitre aisément les locutions prépositives au lieu de devoir en apprendre tout un catalogue. J. CERVONI (1990) se focalise sur l'élaboration d'un ensemble de questionnements problématiques relatifs aux prépositions, auxquels il répond brièvement par la suite avec explication, argumentation et illustration.

\section{Cohérence Vs contradiction}

D. LEEMAN, G. GROSS et J. CERVONI offrent des descriptions cohérentes et consistantes des prépositions et des locutions prépositives. En effet, aucune contradiction, et aucun précepte niant ce qu'un autre soutient, ne sont à souligner.

\section{La validité}

D. LEEMAN, G. GROSS et J. CERVONI proposent des discours valides sur les prépositions et les locutions prépositives. L'analyse de leurs discours montre que l'ensemble des préceptes qu'ils établissent recouvrent généralement les prépositions et les locutions prépositives.

\section{Économie : monosémie vs polysémie}

D. LEEMAN, G. GROSS et J. CERVONI offrent des discours économiques sur le plan de la scientificité linguistique, de par la monosomie des concepts et des mots déployés dans la description de la préposition et des locutions prépositives. Les notions de mot, phrase, groupe de mots, sens stable/ multiple, très usitées par les grammairiens, sont remplacées par d'autres comme morphème, énoncé, syntagme, monosémie/ polysémie, qui sont plus monosémiques. 


\section{Les critères de didacticité}

\section{Modèle global Vs modèle modulaire}

D. LEEMAN et G. GROSS proposent des discours considérablement globaux et exhaustifs sur la préposition et les locutions prépositives, des discours dans lesquels ils relatent parfaitement les fonctions du point décrit et ses caractéristiques sur les plans morphosyntaxique et lexicosémantique. Quant à J. CERVONI, il offre une description très modulaire et fragmentaire des prépositions. Les constituants d'un groupe prépositionnel, les types de prépositions, les caractéristiques syntaxiques et sémantiques des prépositions sont des aspects complètement omis dans son discours.

\section{Intègre du sens / permet de construire du sens}

50 Au niveau du sens, D. LEEMAN et G. CROSS proposent des discours dont l'intégration du sens dans la description des faits est une caractéristique apparente. Ainsi, ils offrent descriptions de la préposition qui excluent toute sorte de formalisme, tout en tenant à joindre des exemples à chaque information fournie. Ces derniers sont souvent incorporés dans l'explication pour servir d'un support d'analyse et d'illustration. J. CERVONI, quant à lui, propose un discours très formel: «[...]une orientation ARB, [...]. La préposition permet l'ajout de la matière notionnelle de $\mathrm{B}$ à celle de $\mathrm{A}$, ajout qui influe sur l'extension discursive de A [...]» (1990: 86). Pour ce qui est de la construction et de la production du sens, D. LEEMAN et G. GROSS vont jusqu'à établir des classes sémantiques afin de mettre en lumière les principaux emplois sémantiques des prépositions (D. LEEMAN, 2008:12) et des locutions prépositionnels (G. GROSS, 2006: 08-09). Par contre, le discours de J. CERVONI ne permet pas de construire ou de produire du sens.

\section{La lisibilité}

51 Au niveau formel, les mots utilisés dans les trois discours sont souvent longs (plus de trois syllabes). Le métalangage déployé est indéfini et abondant, sauf dans le cas de G. GROSS. Quant aux phrases, elles sont considérablement longues (plus de vingt mots) et syntaxiquement complexes. Pour ce qui est de l'image, elle n'est jamais exploitée comme support d'illustration. Au niveau matériel, le critère n'est absolument pas rempli, donc les discours nécessitent une transposition didactique.

\section{L'intelligibilité}

\section{La progressivité}

D. LEEMAN et G. GROSS proposent des discours thématiquement progressifs de par leur démarche descriptive. En effet, en passant par la définition de la préposition, de ses caractéristiques morphologiques et syntaxiques et de ses valeurs sémantiques, ils offrent des discours dont les informations évoluent en crescendo du simple au complexe. Par contre, dans le discours de J. CERVONI, ce critère n'est absolument pas rempli. Son discours forme un continuum d'informations thématiquement éparpillées. 


\section{La parcimonie}

53 Les trois discours reposent sur des discours tellement globaux qu'il devient nécessaire de passer par un grand nombre de concepts et de notions avant d'appréhender le point de langue en question. Cette abondance terminologique ainsi que le nombre des aspects développés dans la description des faits (prépositions, types, morphologie, sémantique, syntaxe, compléments, etc.) privent ces discours de leur didacticité, et par conséquent, le critère de parcimonie n'est absolument pas rempli.

\section{La concrétude}

54 Dans le cas de D. LEEMAN et G. GROSS, les concepts employés sont dans leur totalité abstraits. Mais avec l'intégration du sens dans l'explication des faits, leurs discours deviennent plus ou moins concerts. Ainsi, ces concepts sont dans la majorité des cas incarnés et matérialisés à travers des exemples et des illustrations. Par contre, dans le discours de J. CERVONI (1990: 86), ce critère n'est absolument pas rempli. Les prépositions sont abordées comme des constructions mentales sans aucun souci d'illustration.

\section{Emploi d'images et de métaphores incarnant la vie quotidienne de l'apprenant}

Aucun des linguistes ne recourt à l'emploi de supports visuels, de métaphores ou de métonymies pour mieux illustrer leurs descriptions, ce qui assigne un caractère particulièrement théorique et explicatif à leurs discours. Donc le critère n'est pas rempli.

A présent, nous tâcherons de résumer ci-dessous, moyennant la grille d'analyse de la valeur d'applicabilité (M-E DAMAR, 2009), les résultats de notre examen de la scientificité linguistique et de la didacticité des grammaires Le Bescherelle (A), Le Larousse (B) et Le Robert \& Nathan (C) et des discours de D. LEEMAN (D), de D. GROSS (E) et de J. CERVONI (F). 
Tableau 1 : Résultats de l'analyse des discours grammaticaux et linguistiques

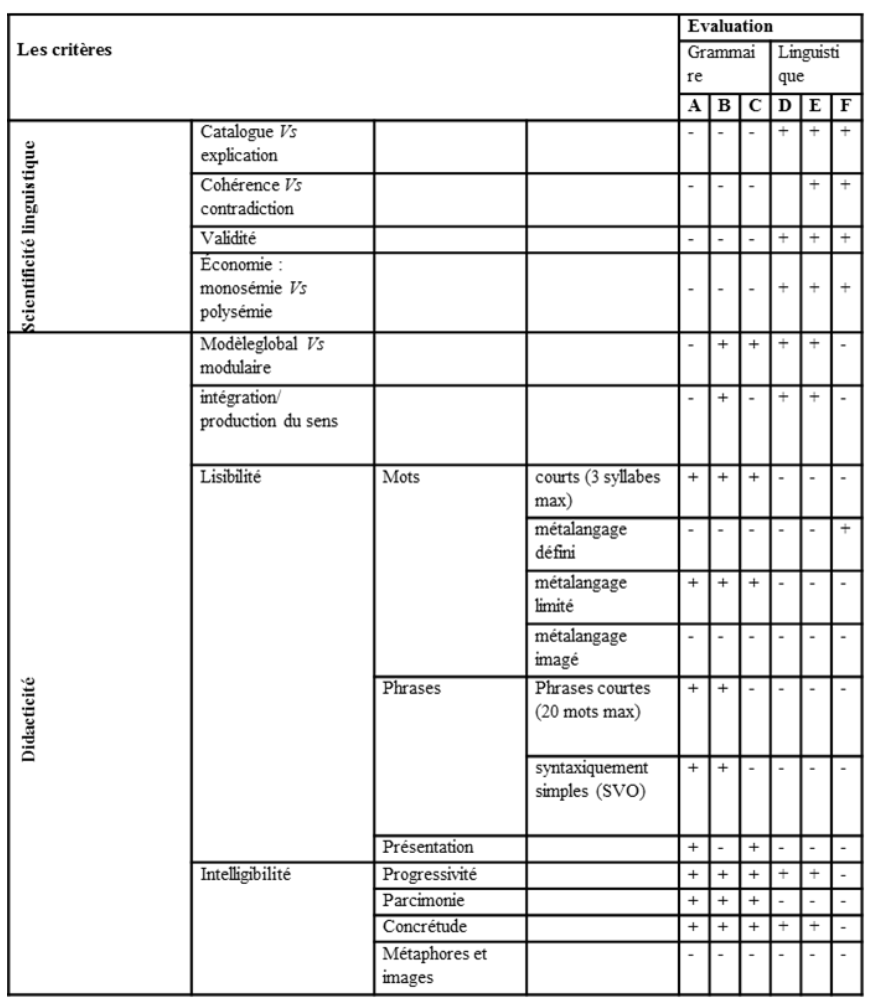

\section{Synthèse}

L'examen de l'ensemble des discours nous a permis de constater qu'aucune des grammaires analysées ne remplit les critères de scientificité (0/4). Sur le plan didactique, Le Bescherelle offre un discours grammatical plus ou moins didactique de par sa lisibilité, sa progressivité, sa parcimonie et sa concrétude (3/7). Le Larousse remplit tous les critères de didacticité excepté l'emploi d'image et de métaphores (4/7). Le Robert \& Nathan ne satisfait que le critère de globalité, de progressivité et concrétude (2/7). Quant aux discours linguistiques, D. LEEMAN propose un discours linguistiquement scientifique (4/4) et didactiquement global, sémantique, progressif et concret (4/7). Le discours de D. GROSS est scientifique d'un point de vue linguistique (4/4) et global, sémantique, progressif et concret d'un point de vue didactique (4/7). Le discours de J. CERVONI est scientifique sur le plan linguistique (3/4), il satisfait aussi, sur le plan didactique, le critère de définition du métalangage (0/7).

Le graphique ci-dessous constitue un aperçu global et synthétique des critères les plus rencontrés dans les grammaires et dans les discours linguistiques. Une simple observation permet en effet de constater que les critères de scientificité linguistique sont exclusivement remplis par les discours linguistiques. Les discours grammaticaux, quant à eux, satisfont le plus les critères de lisibilité, de progressivité et de concrétude. Dès lors, nous pouvons avancer que les discours linguistiques nécessitent une transposition didactique pour remporter leur enseignabilité alors que ceux didactiques requièrent un adjuvant pour surpasser leurs lacunes et mériter leur transposabilité. En effet, une didactisation des contenus linguistique pourrait fructueusement contribuer à 
l'amélioration de la tâche enseignement/ apprentissage de la grammaire. La conjugaison des apports des deux disciplines permettra ainsi donner lieu à un enseignement/ apprentissage de grammaire plus fructueux de par la qualité de ses contenus et la forme dont ils sont présentés. A ce niveau, le discours linguistique est envisagé comme objet d'enseignement et non pas comme outil au service de l'enseignement.

Figure 1 : Les crières les plus rencontrés dans les deux types de discours

Il va sans dire que dans une perspective didactique, une transposition didactique s'avère d'une nécessité capitale dans tout enseignement/ apprentissage. Considérée comme étant une adaptation d'un savoir brut au profil de l'apprenant, ou encore comme un passage d'un savoir scientifique à un autre dit didactique, cette opération ne saurait en aucune manière évaluer la qualité des savoirs à enseigner. Un savoir devrait, à notre sens, être transposable avant d'être transposé, être enseignable avant d'être enseigné. Nous passerons alors d'une perspective de transposition à une perspective de transposabilité pour parler ainsi d'une linguistique plutôt applicable à l'enseignement et non pas appliquée, c'est-à-dire, de la pertinence d'un discours linguistique d'être impliquée dans une situation d'enseignement/ apprentissage. A ce niveau, le discours linguistique est envisagé comme objet d'enseignement et non comme outil au service de l'enseignement. De ce fait, nous pensons que la valeur d'applicabilité est le moyen le plus efficace pour concrétiser cette fructueuse aide que la linguistique pourrait concéder à la didactique de la grammaire.

\section{Conclusion}

L'objectif de la présente contribution n'est nullement de remettre en question la grammaire comme discipline mais plutôt d'attester des lacunes et des défaillances qu'elle présente, et de mettre l'accent sur la nécessité impérieuse de repenser la réconciliation de la didacticité de la grammaire et de la scientificité de la linguistique. Les critères transgressés par les discours grammaticaux au niveau de leur scientificité linguistique constituent, en réalité, les bases solides des discours linguistiques qui, à leur tour, sont sujets à caution sur le plan didactique. De ce fait, la conciliation systématique entre la scientificité des recherches linguistiques (explication, cohérence, validité et économie) et la didacticité des grammaires (lisibilité, progressivité et concrétude) devrait sans doute permettre de fonder des contenus grammaticaux aussi bien scientifiques que didactiques, scientifiques de par leur qualité et didactiques de par leur forme. Ainsi, on serait en mesure de remédier aux lacunes de la première et de mettre fin à la stérilité de la deuxième. 


\section{BIBLIOGRAPHIE}

BOUVERESSE, R. (1978). KARL POPPER ou Le rationalisme critique. Paris : Librairie philosophique J. VRIN.

CANELAS-TREVISI, S. (2009). La grammaire enseignée en classe, le sens des objets et des manipulations. Bruxelles : Peter Lang,

CERVONI, J. (1990). « Prépositions et compléments prépositionnels ». Revue Langue française, $\mathrm{n}^{\circ} 01$. pp. 85-89. http://www.persee.fr/doc/lfr_0023-8368_1990_num_86_1_5795

CHISS, J-L. (2016). De la pédagogie du français à la didactique des langues : les disciplines, la linguistique et l'histoire. France : L'édition de l'école polytechnique.

CHRISTENSEN, M-H. FUCHS, M. KORACH, D. \& SCHAPIRA, C. (1995). Les guides Robert et Nathan : Grammaire. France : Nathan.

CUQ, J.-P. (1996). Une introduction à la didactique de la grammaire en français langue étrangère. Paris : Didier.

CUQ, J-P. (2003). Dictionnaire de didactique du français langue étrangère et seconde. Paris : CLE International.

DAMAR, M.-E. (2009). « La valeur d'applicabilité d'une théorie linguistique, l'exemple des articles un et le en français ». Revue Les Cahiers de l'Acedle, n 02. pp. 101-128. https://acedle.org/old/IMG/ pdf/Damar-M--E--La_valeur_d_applicabilite_d_une_theorie_linguistique.pdf

DAMAR, M.-E. (2009). Pour une linguistique applicable, L'exemple du subjonctif en FLE. Bruxelles : Peter Lang.

DAMAR, M.-E. (2010). « La valeur d'applicabilité d'une théorie linguistique, une interface entre linguistique et didactique, l'exemple du subjonctif en FLE ». Bruxelles : Peter Lang, pp. 113-135.

DUBOIS, J. \& LAGANE, R. (1995). Larousse Grammaire. Espagne : Larousse.

DUBOIS, J. (1999). Dictionnaire de linguistique et des sciences du langage. Paris : Larousse.

GIRARD, D. (1972). Linguistique appliquée et didactique des langues. Paris : Armand Colin_ Longman.

GREVISSE, G. \& GOSSE, A. (2008). Le bon usage (14 ${ }^{\text {ème }}$ éd). Bruxelles : De Boeck \& Larcier.

GROSS, G. (2006). « Sur le statut des locutions prépositives », Revue Modèles linguistiques, nº 53.

pp. 01-14. http://ml.revues.org/517

KALMBACH, J.-M. (2008). « Intégrer les marqueurs d'infinitif dans la grammaire française ». Revue Synergie pays scandinaves, $\mathrm{N}^{\circ}$ 03. pp. 63-74 http://gerflint.fr/Base/Paysscandinaves3/ kalmbach.pdf

LAURENT, N. \& DELAUNAY, B. (2012), Bescherelle, La grammaire pour tous. Paris : Hatier.

LEEMAN, D. (2008). « Prépositions du français : état des lieux ». Langue française, $\mathrm{n}^{\circ}$ 157. pp. 05-19. http://www.cairn.info/revue-langue-francaise-2008-1-page-5.htm

MARTINET, A. (2005). Eléments de linguistique générale. Paris : Armand Colin. 
PUREN, CH. (2008). « La didactique scolaire des langues à l'ère pré-applicationniste : Grammaire et linguistique dans les années 1880-1890 ». Revue Synergie Algérie, n 02. pp. 35-54. https:// gerflint.fr/Base/Algerie2/puren.pdf

RIEGEL, M., PELLAT, J-C. \& RIOUL R.(2009).Grammaire méthodique du français. Paris : PUF.

\section{NOTES}

1. Voir à ce sujet M-E. Damar (2009), M-E. Damar (2010)

2. Voir à ce sujet Ch. PUREN (1988) et J-P CUQ (1996).

3. Tous les critères cités seront définis au fur et à mesure de notre progression dans l'analyse de notre corpus.

\section{RÉSUMÉS}

Cet article se propose de repenser la possibilité d'exploiter les apports de la linguistique fondamentale à des fins didactiques, en particulier, l'enseignement/ apprentissage de la grammaire du FLE. La comparaison de la qualité/ la forme des contenus proposés par les discours grammaticaux et les discours linguistiques quant à la description des prépositions de la langue française nous a permis de déceler les lacunes caractérisant les grammaires dans leur description des points de langue. Nous mettrons l'accent dans un premier temps sur la scientificité linguistique et la didacticité de trois des grammaires les plus usitées dans l'enseignement/ apprentissage du FLE en Algérie. Dans un second temps, nous tâcherons de faire de même avec trois discours linguistiques afin de mettre en lumière la possibilité de développer une réflexion sur une grammaire aussi bien scientifique sur le plan linguistique que pédagogique en classe de FLE.

This article proposes to rethink the possibility of exploiting the contributions of fundamental linguistics for didactic purposes, in particular, teaching grammar of the French as a foreign language (FFL). Comparing the linguistic scientificity / didacticity of grammatical and linguistic discourses as to the description of the prepositions of the French language allowed us to highlight the gaps characterizing grammars in their description of the grammatical facts. We shall focus first on the linguistic scientificity and the didacticity of three most frequently used grammars in the teaching / learning of the grammar of FFL in Algeria. In a second step, we will try to do the same with three linguistic theories in order to highlight the possibility of developing a thinking on a scientific and a pedagogical grammar in class of FFL.

\section{INDEX}

Mots-clés : grammaire, linguistique, didactique, enseignement du FLE, prépositions

Keywords : grammar, linguistic, didactics, French as a foreign language, preposition 


\section{AUTEURS}

\section{ASSIAKH SOFIANE}

Laboratoire LESMS, Faculté des lettres et des langues, Université de Bejaia, 06000 Bejaia, Algérie, ASSIAKH Sofiane est doctorant en $4{ }^{\text {ème }}$ années (Option : linguistique appliquée à l'enseignement du FLE) et enseignant au département de français, faculté des lettres et des langues, Université de Bejaia, Algérie. Il est aussi membre du laboratoire LESMS. Il travaille sur l'enseignement/ apprentissage de la grammaire en classe de FLE. Il est titulaire d'une licence en Sciences du langage et Didactique et d'un master en Science du langage.

Courriel : Assiakh_sofiane[at]yahoo.fr

\section{SADI NABIL}

Laboratoire LESMS, Faculté des lettres et des langues, Université de Bejaia, 06000 Bejaia, Algérie, SADI Nabil est docteur en Sciences et maitre de conférences en Sciences du langage au département de français, faculté des lettres et des langues, Université de Bejaia, Algérie. Il est aussi directeur du laboratoire LESMS. Il travaille principalement sur le français parlé dans les médias algériens, sur la variation et le style en milieux plurilingues. Il est l'auteur d'une quinzaine de publications.

Courrier : sadinabil[at]hotmail.com 